\title{
DETECTION OF TRAFFIC CONGESTION IN OPTICAL REMOTE SENSING IMAGERY
}

\author{
Gintautas Palubinskas, Franz Kurz and Peter Reinartz \\ German Aerospace Center DLR \\ Remote Sensing Technology Institute \\ Münchener Str. 20, 82234 Wessling, Germany \\ Gintautas.Palubinskas@dlr.de
}

\begin{abstract}
A new approach for the traffic congestion detection in time series of optical digital camera images is proposed. It is well suited to derive various traffic parameters such as vehicle density, average vehicle velocity, beginning and end of congestion, length of congestion or for other traffic monitoring applications. The method is based on the vehicle detection on the road segment by change detection between two images with a short time lag, the usage of a priori information such as road data base, vehicle sizes and road parameters and a simple linear traffic model based on the spacing between vehicles. The estimated velocity profiles for experimental data acquired by airborne optical remote sensing sensor - 3K camera system - coincide quite well with the reference measurements.
\end{abstract}

Index Terms - Optical remote sensing, time series images, congestion, change detection, traffic model

\section{INTRODUCTION}

During the past years, increasing traffic appears to be one of the major problems in urban and sub-urban areas [1]. Traffic congestion and jams are one of the main reasons for immensely increasing transportation costs due to the wasted time and extra fuel. A new type of information is needed for a more efficient use of road networks. Remote sensing sensors installed on aircrafts or satellites enable data collection on a large scale and thus seem to be very suitable for various traffic monitoring applications. Several airborne optical remote sensing systems are already in experimental use at German Aerospace Center DLR, e.g. airborne 3K camera system [2], consisting of three digital cameras capable of acquiring three images per second, and LUMOS [3]. Automatic detection of vehicles and estimation of their velocities in sequences of optical images is still a challenge and at present still results in a too low completeness (less than $70 \%$ ) thus being not yet suitable e.g. for the estimating of the traffic density.

\section{METHOD}

We approach the traffic congestion detection in sequences of optical images in a different way. We don't try to detect each individual vehicle and then to estimate its velocity as e.g. in [4], but instead of that we model a traffic flow on the road segment and thus derive directly the required traffic parameters from the data. Thus, our traffic congestion detection method is based on the combination of various techniques: change detection, image processing and incorporation of a priori information such as traffic model and road network. The change detection in two images with a short time lag is implemented using the recently proposed change detection method [5] - Multivariate alteration detection (MAD) - resulting in a change image where the moving vehicles on the roads are highlighted. Image processing techniques can be applied to derive the vehicle density in the binarized change image. This estimated vehicle density can be related to the theoretical vehicle density, which can be acquired by modeling the traffic flow for a road segment. The model is derived from a priori information about the vehicle sizes and road parameters [6], the road network, e.g. NAVTEQ road data base [7], and the spacing between the vehicles. This theoretical vehicle density is directly related to the average vehicle velocity on the road segment [8] and thus the information about the traffic situation, e.g. the existence of congestion, the beginning and end of congestion, the length of jam, actual travel times, and so on can be derived. The flow diagram of the proposed method is shown in Fig. 2.

\subsection{Vehicle detection}

Detection of vehicles on the road segment is performed in the following way. First, two images with a short time lag e.g. 3 seconds (derived from two constraints: vehicle should not overlap with itself and with the previous vehicle) are selected, then the region of interest (tube) is defined based on the road line and change image is obtained with a MAD algorithm. Finally, the obtained change image - chi squared image of MAD components - is binarized and denoised, e.g. by median filter. Now the vehicle density can be 
estimated for each road segment from the binarized image defined as a ratio of the number of white pixels to the total number of pixels in the road segment.

\subsection{A priori information}

The following a priori information: road data base (road lines in two directions), vehicle sizes for passenger cars and trucks and road parameters (number of lanes, lane width) is used in the proposed method.

\subsection{Traffic model}

A simple linear traffic model is proposed

$$
S=B \cdot g(v)+L,
$$

where spacing $S$ is front-to-front vehicle distance in meter, $B$ is a dimensionless parameter of the model, function $g(v)$ transforms velocity $(\mathrm{km} / \mathrm{h})$ into meters e.g. $g(100 \mathrm{~km} / \mathrm{h})=100 \mathrm{~m}$ and $L$ is vehicle length in meter. Parameter $B$ can be interpreted in the following way: for $B=0.5$ and $L=0$ the formula (1) means for all drivers wellknown rule of thumb "safe distance $=$ half speedometer reading in metres". For a congested traffic $B$ value is ranging between 0.5 and 1.0. Now the theoretical vehicle density can be calculated as

$$
D(\# \text { vehicle per } \mathrm{km})=\frac{1 \mathrm{~km}}{\mathrm{~S}} \text {. }
$$

In this formula intrinsic assumptions about the constant speed and same type of vehicles for a selected road segment are implied.

\section{EXPERIMENT}

To confirm our idea and to validate the method several flight campaigns with the DLR airborne experimental wide angle optical 3K digital camera system operated on a Do228 aircraft were performed during the recent years. In this paper one of such experiments is presented.

\subsection{DLR 3K camera system}

The 3K camera system (“3 Kopf” = "3 head”) consists of three non-metric off-the-shelf cameras (Canon EOS 1Ds Mark II, 16 MPix). The cameras are arranged in a mount with one camera looking in nadir direction and two in oblique sideward direction, which leads to an increased FOV of max $110^{\circ} / 31^{\circ}$ in across track/flight direction. The camera system is coupled to a GPS/IMU navigation system, which enables the direct georeferencing of the 3K optical images. Fig. 1 illustrates the image acquisition geometry of the DLR 3K camera system. Based on the use of $50 \mathrm{~mm}$ Canon lenses, the relation between airplane flight height, ground coverage, and pixel size is shown, e.g. the pixel size at a flight height of $1000 \mathrm{~m}$ above ground is $15 \mathrm{~cm}$ and the image array covers up $2.8 \mathrm{~km}$ in width.

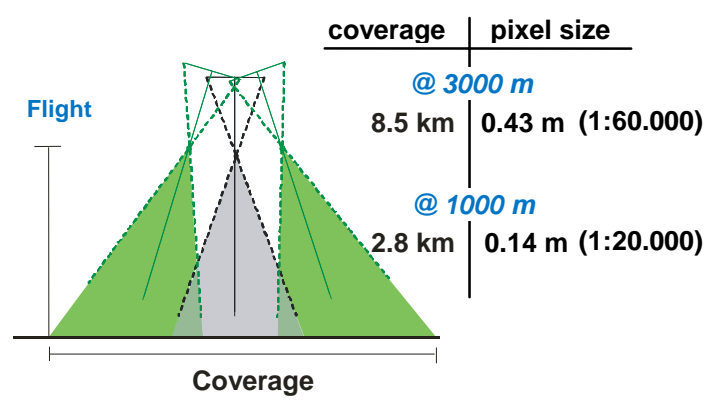

Fig 1 Illustration of the image acquisition geometry for 3K camera system. The tilt angle of the sideward looking cameras is approx. $35^{\circ}$.

\subsection{Test site and data}

The motorway A8 south of Munich is one of the busiest parts of the German motorway network with an average load of around 100.000 vehicles per day. Test site was a $16 \mathrm{~km}$ motorway section between motorway junctions "Hofolding” and "Weyarn". On $2^{\text {th }}$ Sep. 2006, heavy traffic was expected at this section caused by homebound travellers. Three 3K data takes were acquired between 14:01 and 15:11 from $2000 \mathrm{~m}$ above ground in three overflights. During each overflight, 22 image bursts were acquired each containing four consecutive images. The time difference within these bursts was $0.7 \mathrm{~s}$, so that each car was monitored at least $2.1 \mathrm{~s}$.

\subsection{Results}

Results of the traffic congestion detection on the test site are shown in Fig. 3. In this figure, (a) is the first image of an example sequence, (b) - the second image acquired 2.1 seconds later, (c) - binarized chi squared image of MAD components after change detection, (d) - velocity profiles for separate road directions plotted on the change detection image and (e) - reference velocity measurements plotted on the first image. In red color the congestion area is marked exhibiting average velocity up to $80 \mathrm{~km} / \mathrm{h}$ what corresponds quite well with reference data acquired simultaneously.

\section{DISCUSSION}

Experimental results show the potential of the proposed method for the detection of traffic congestion on highways in along-track scenes. The following conclusions can be drawn. For the accurate vehicle density estimation the optimal time lag between the two images seems to be about 3 seconds and the range of the velocities between $10 \mathrm{~km} / \mathrm{h}$ and $80 \mathrm{~km} / \mathrm{h}$ (congested traffic situation). The problem 
occurring for very slow or stopped vehicles can be solved by incorporating other approaches, e.g. classification of the single image. Distortions due to vehicle shadows and off nadir acquisitions can be minimized by using additional a priori information such as sun elevation and vehicle height. In total, the method produces quite stable and robust results even for short road segments of up to 100 meters. The accuracy of produced parameter estimates seems to be sufficient, but needs to be investigated quantitatively.

\section{CONCLUSION}

A new traffic congestion detection approach for time series images acquired by airborne optical 3K camera system is introduced. It allows us to derive various traffic parameters such as vehicle density, average velocity, beginning and end of congestion, length of congestion and so on. The method is based on the vehicle detection on the road segment by change detection of two images with a short time lag, usage of a priori information and a simple traffic model. The estimated velocity profiles coincide quite well with the reference measurements.

\section{ACKNOWLEGMENT}

The authors would like to thank colleagues Erich Bogner and Rolf Stätter for their efforts in planning the flight campaign, data acquisition and data processing. Special thanks to our trainee Mantas Palubinskas for the implementation and validation of the traffic model.

\section{REFERENCES}

[1] http://ops.fhwa.dot.gov/congestion_report/ (Accessed on June 13, 2008).

[2] F. Kurz, R. Müller, M. Stephani, P. Reinartz, and M. Schroeder, "Calibration of a wide-angle digital camera system for near real time scenarios”, Proc. ISPRS Hannover Workshop 2007 High Resolution Earth Imaging for Geospatial Information, Hannover, Germany, May 29 - June 1, 2007.

[3] I. Ernst, S. Sujew, K.-U. Thiessenhusen, M. Hetscher, S. Raßmann, and M. Ruhé, "LUMOS - airborne traffic monitoring system", Proceedings of 6th IEEE International Conference on Intelligent Transportation Systems, Shanghai, China, 2003.

[4] P. Reinartz, M. Lachaise, E. Schmeer, T. Krauß, and H. Runge, "Traffic Monitoring with Serial Images from Airborne Cameras", ISPRS Journal of Photogrammetry and Remote Sensing, vol. 61, pp. 149-158, 2006.

[5] A. A. Nielsen, "The Regularized Iteratively Reweighted MAD Method for Change Detection in Multi- and Hyperspectral Data", IEEE Transactions on Image Processing, vol. 16, no. 2, pp. 463478, 2007.

[6] http://www.sicherestrassen.de/VKO/QuerschnitteFahrbahn.htm (Accessed on June 13, 2008).

[7] http://www.navteq.com/about/data.html (Accessed on June 13, 2008).

[8] K.-M. Kockelman, "Changes in the Flow-Density Relation due to Environmental, Vehicle, and Driver Characteristics", Transportation Research Record, 1644, pp. 47-56, 1998.

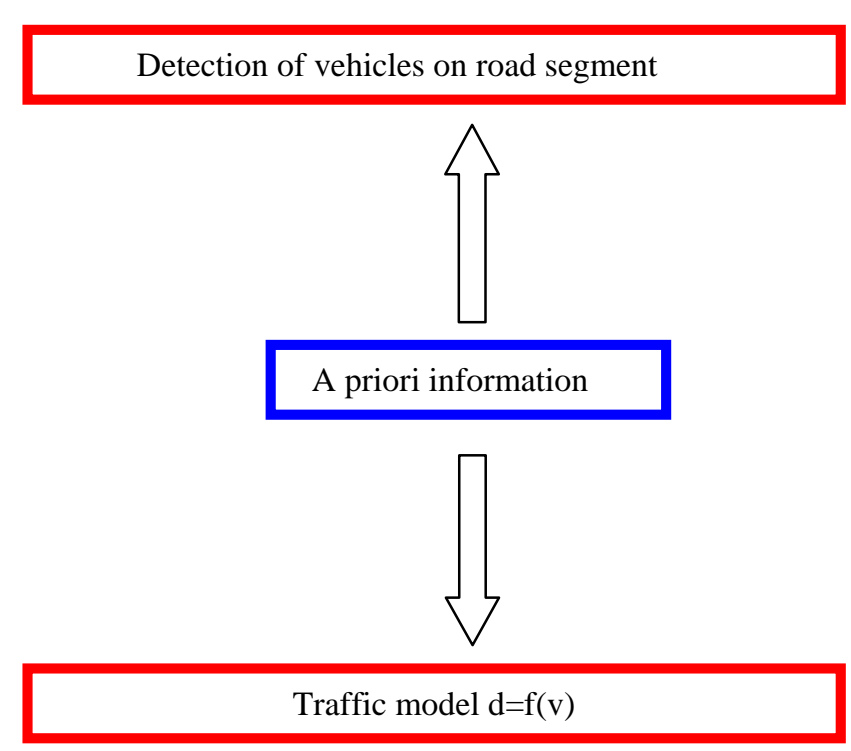

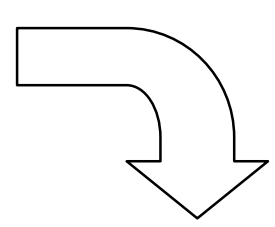

Estimated vehicle density $d$

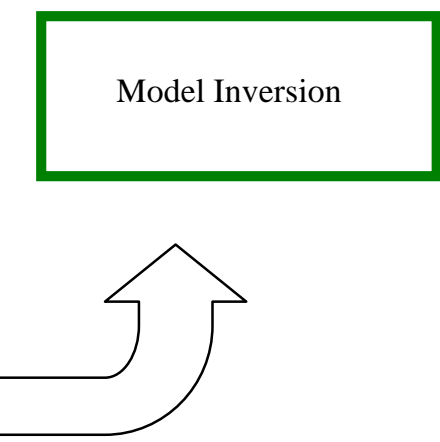

Average velocity per road segment $v$

Fig. 2 Flow diagram of the proposed traffic congestion detection method. 


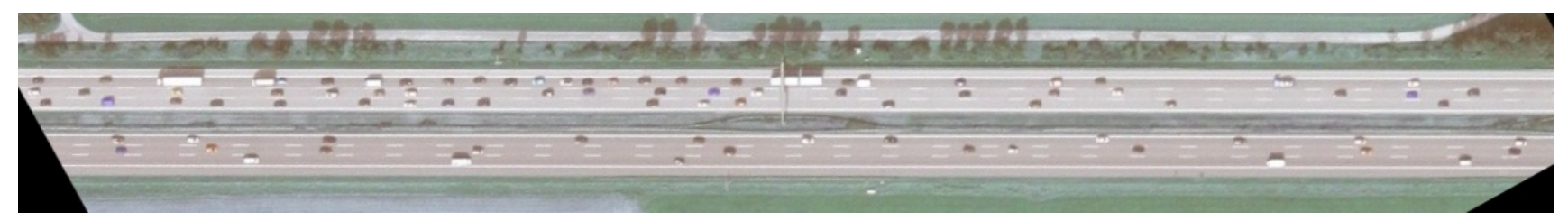

a

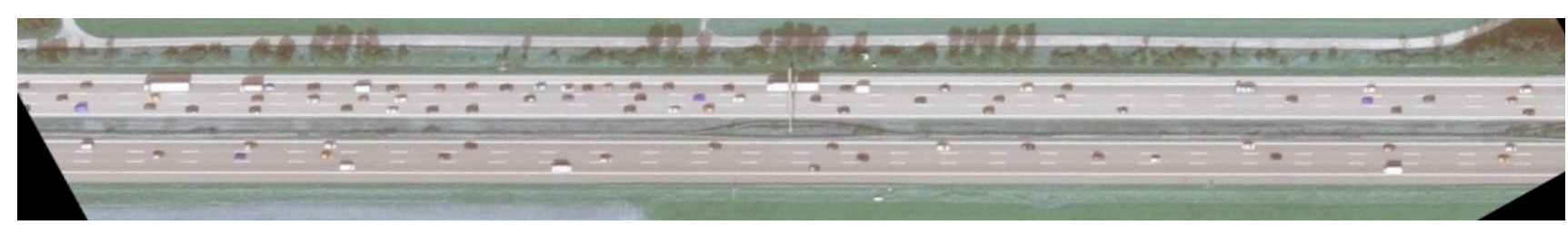

b

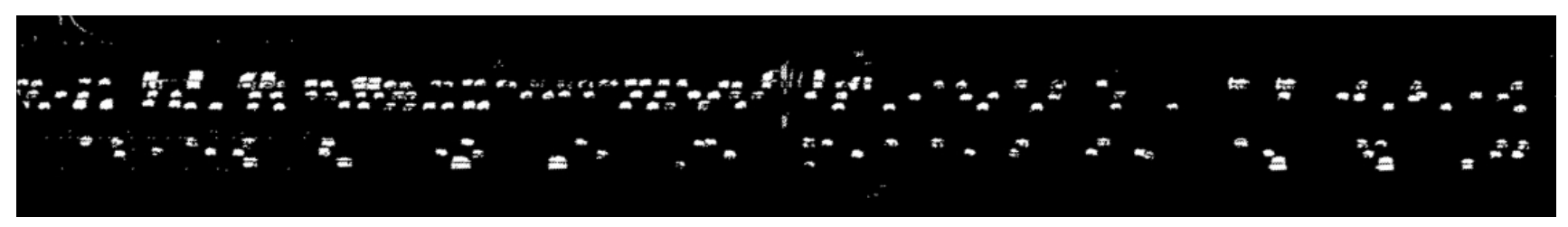

C

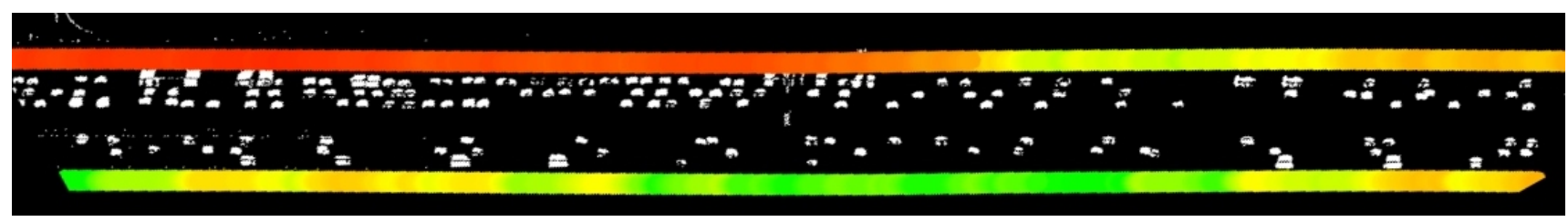

d

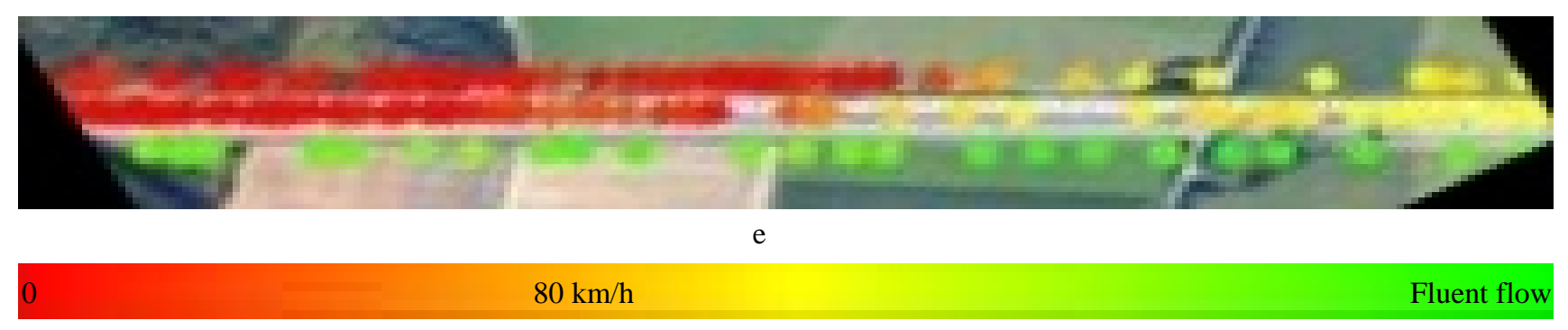

Fig. 3 Example of the traffic congestion detection on A8 highway between Munich and Salzburg for 3K sensor data acquired during ADAC flight campaign on 2.9.2006: (a) first original image, (b) second image acquired 2.1 seconds later, (c) binarized chi squared image of MAD components after change detection, (d) velocity profiles for separate road directions plotted on the change detection image and (e) reference velocity measurements plotted on the original image. In red color the congestion area is marked exhibiting average velocity up to $80 \mathrm{~km} / \mathrm{h}$ what corresponds quite well with reference data acquired simultaneously. 